\title{
Static versus dynamic analysis of the influence of gravity on concentration non-equilibrium fluctuations ${ }^{\star}$
}

\author{
Fabrizio Croccolo ${ }^{1,2, a}$, Henri Bataller ${ }^{1}$, and Frank Scheffold ${ }^{2}$ \\ 1 Laboratoire des Fluides Complexes et leurs Réservoirs - UMR5150, Université de Pau et des Pays de l'Adour, 64600 Anglet, \\ France \\ 2 Department of Physics, University of Fribourg, CH-1700 Fribourg, Switzerland
}

Received 26 June 2014 and Received in final form 2 September 2014

Published online: 6 November 2014 - (C) EDP Sciences / Società Italiana di Fisica / Springer-Verlag 2014

\begin{abstract}
In a binary fluid mixture subject to gravity and a stabilizing concentration gradient, concentration non-equilibrium fluctuations are long-ranged. While the gradient leads to an enhancement of the respective equilibrium fluctuations, the effect of gravity is a damping of fluctuations larger than a "characteristic" size. This damping is visible both in the fluctuation power spectrum probed by static and the temporal correlation function probed by dynamic light scattering. One aspect of the "characteristic" size can be appreciated by the dynamic analysis; in fact at the corresponding "characteristic" wave vector $q^{*}$ one can observe a maximum of the fluctuation time constant indicating the more persistent fluctuation of the system. Also in the static analysis a "characteristic" size can be extracted from the crossover wave vector. According to common theoretical concepts, the result should be the same in both cases. In the present work we provide evidence for a systematic difference in the experimentally observed "characteristic" size as obtained by static and dynamic measurements. Our observation thus points out the need for a more refined theory of non-equilibrium concentration fluctuations.
\end{abstract}

\section{Introduction}

Fluctuating hydrodynamics (FHD) is widely used to describe the statistical properties of non-equilibrium fluctuations (NEFs) in a variety of configurations [1]. A number of approximations can be included in the theory in order to obtain analytical solutions and several refinements of the basic theory have been reported in the literature [2-4]. The theoretical results have also been tested and largely confirmed by advanced simulation tools $[5,6]$. The main results of FHD are the predictions made for NEFs, such as the static power spectrum, and the temporal correlation function, both as a function of the wave vector. These quantities are directly accessible by means of static and dynamic light scattering and much experimental effort has been devoted over the last decades to the verification and testing of the available theoretical models [1]. More recently this analysis has also been employed to measure the transport properties of binary liquid mixtures like Soret and mass diffusion coefficients $[7,8]$. Moreover two remarkable experiments on non-equilibrium fluctuations

\footnotetext{
* Contribution to the Topical Issue "Thermal nonequilibrium phenomena in multi-component fluids" edited by Fabrizio Croccolo and Henri Bataller.

a e-mail: fabrizio.croccolo@univ-pau.fr
}

have been performed in space under microgravity conditions $[9,10]$. While theoretical predictions have generally been confirmed and the impact of gravity has been studied experimentally both on the statics $[11,12]$ and on the dynamics $[7,8,13,14]$ of NEFs, in this paper we point out a small but systematic difference between the "characteristic" wave vector $q^{*}$ as obtained from the two different experimental approaches. The latter points out the need for a more refined theory of concentration NEFs. An attempt to include realistic boundary effects, or the effect of confinement, has been recently made [15] and its impact is discussed here.

The paper is organized as follows: Section 2 provides a short introduction of the theory of concentration NEFs (c-NEFs), sect. 3 describes the experimental procedure of the reported experiments (from previous literature), sect. 4 shows the comparison between the extracted values of $q^{*}$ as obtained from the statics and dynamics, finally in sect. 5 conclusions are reported.

\section{Non-equilibrium fluctuations}

Every system exhibits fluctuations of its thermodynamic variables even under equilibrium conditions. The intensity of equilibrium fluctuations is independent of their size and 
can be described as a white noise [16]. When a macroscopic temperature or concentration gradient is applied to the system NEFs appear and their spectrum becomes strongly dependent on their size due to a coupling between the temperature or concentration fluctuations and the velocity ones; the coupling being driven by the presence of the gradient. In the presence of gravity the resulting density gradient can be either stabilizing or destabilizing depending on the relative orientation of the density gradient and gravity so that the stability of the fluid can be quantitatively expressed by means of the Rayleigh number in its thermal or solutal formulation:

$$
\begin{aligned}
& R a_{T}=-\frac{\alpha \vec{g} \cdot \vec{\nabla} T}{\nu \kappa} L^{4}=-\frac{\vec{g} \cdot \vec{\nabla} \rho_{T}}{\rho \nu \kappa} L^{4}, \\
& R a_{s}=-\frac{\beta \vec{g} \cdot \vec{\nabla} c}{\nu D} L^{4}=-\frac{\vec{g} \cdot \vec{\nabla} \rho_{s}}{\rho \nu D} L^{4},
\end{aligned}
$$

where $\alpha=(1 / \rho)(\partial \rho / \partial T)$ is the thermal expansion coefficient, $\vec{g}$ the gravitational acceleration, $\vec{\nabla} T$ the temperature gradient, $\nu$ the kinematic viscosity, $\kappa$ the thermal diffusion coefficient, $L$ the vertical extension of the sample, $\vec{\nabla} \rho_{T}$ the thermally generated density gradient, $\beta=(1 / \rho)(\partial \rho / \partial c)$ the solutal expansion coefficient of the denser component, $\vec{\nabla} c$ the gradient of the concentration of the denser component, $\vec{\nabla} \rho_{s}$ the solutal density gradient and $D$ the mass diffusion coefficient. The macroscopic gradient has thus a "stabilizing" (respectively, "destabilizing") effect if the corresponding Rayleigh number is negative (positive).

It can be shown that a similar description can be obtained for thermal or concentration non-equilibrium fluctuations (c-NEFs) by simply substituting thermal with solutal parameters in the relevant equations. A remarkable difference is however obtained if confinement effects are taken into account, but their impact is negligible in the present work [17]. In the remainder of this paper only concentration fluctuations will be taken into account. Moreover we only consider the case when the density gradient is "stabilizing" and in the following we will always assume negative Rayleigh numbers and mention their absolute values.

In our relevant wave vector range, c-NEFs evolve over time mainly driven by two effects: on the one side mass diffusion given by the presence of the concentration gradient and on the other side gravity due to the presence of the density gradient. The key feature is then the fluctuation size $\lambda$ (or, inversely, its wave vector $q=2 \pi / \lambda$ ) because for different sizes either of the two effects dominates. For instance, should the fluctuation be greater than a "characteristic" size (small wave vectors) then diffusion happens to be a slow process and therefore gravity can be the dominant one. On the contrary, if the fluctuation is smaller than the mentioned "characteristic" size (large wave vectors) then diffusion is the faster dominating process. Available theories allow us to define the solutal "characteristic" wave vector [18] as

$$
q_{s}^{*}=\left(\frac{\beta g \nabla c}{\nu D}\right)^{1 / 4}
$$

where $\nabla c$ is the modulus of the concentration gradient.

Comparing eq. (2) and (3) we note that the "characteristic" wave vector is closely related to the Rayleigh number of the fluid mixture:

$$
\left|R a_{s}\right|=\left(q_{s}^{*} L\right)^{4} .
$$

Analysis of the c-NEF dynamics $[4,7,8,13,14]$ reveals that the time constant of fluctuations is the classical diffusive time constant $\tau=1 /\left(D q^{2}\right)$ for values $q \gg q_{s}^{*}$ while it quadratically increases as a function of the wave vector $\tau \propto q^{2}$ for values $q \ll q_{s}^{*}$ showing a clear maximum at $q=q_{s}^{*}$ thus identifying the most persistent fluctuation of the system. More precisely the time constant can be written as:

$$
\tau=\frac{1}{D q^{2}\left[1+\left(q_{s}^{*} / q\right)^{4}\right]} .
$$

On the other side, the static power spectrum of c-NEFs is characterized by a strong $q^{-4}$ divergence for $q \gg q_{s}^{*}$ and a plateau for $q \ll q_{s}^{*}$, the resulting function having a roll-off for the same value $q=q_{s}^{*}$. The complete equation can be written as:

$$
I_{S}(q)=\frac{I_{o}}{1+\left(q / q_{s}^{*}\right)^{4}},
$$

where $I_{o}=\left[k_{B} T /\left(16 \pi^{4} \rho\right)\right] \times\left[|\nabla c| /(\beta g)-(\partial c / \partial \mu)_{p, T}\right]$ is the value of the plateau for wave vectors $q \ll q_{s}^{*}, k_{B}$ the Boltzmann constant, $T$ the average temperature of the mixture, $\mu$ the chemical potential per unit mass and $p$ the pressure.

Typical values of $q_{s}^{*}$ in molecular binary mixtures are of the order of $100 / \mathrm{cm}$, not accessible to classical light scattering techniques. A comparison of the theoretical predictions on the effect of gravity with an experiment only became a possibility with the advent of optical techniques able to investigate the appropriate wave vector range. As a matter of fact, the first measurements of c-NEFs have been reported in 1993 by means of dynamic light scattering [19] and at that time it was not possible to observe the effect of gravity at small values of $q$. Interestingly, in that paper the authors suggested for the first time the possibility of measuring the Soret coefficient from the investigation of c-NEFs. The first measurements of the static power spectrum of c-NEFs including the observation of the effect of gravity was performed some years later using ultra low angle static light scattering (USLS) [11, 12]. Here the authors investigated a critical binary mixture with a relatively "large" crossover value of $q_{s}^{*}$ on the order of $400-1000 / \mathrm{cm}$ and more. The first direct evidence for the existence of a relaxation time maximum in a dynamic measurement required the development of novel dynamic near-field scattering (d-NFS) techniques such as dynamic Shadowgraph and Schlieren $[13,14]$ to provide reliable measurements in the proximity of and below $q_{s}^{*}$ for ordinary binary mixtures. 


\section{Experimental procedure}

In this work we expand on two experiments published recently $[7,8,13,14]$ : Soret-driven experiments, where a temperature gradient is applied to an initially homogeneous binary mixture to get a concentration gradient by means of the Soret effect, and free-diffusion experiments where an isothermal liquid is put on top to a denser one at the same temperature and the system is let evolve by mass diffusion only towards the equilibrium homogeneous state.

Soret-type experiments have been performed by imposing a temperature gradient to a horizontal slab filled with a fluid. In order to obtain a precise thermal control the fluid is confined horizontally by an O-ring and vertically by two sapphire plates allowing both a good heat transfer and an optical access to the liquid. The two sapphires are kept at a distance $L=0.153 \mathrm{~cm}$ or $L=0.130 \mathrm{~cm}$ for the reported experiments by four plastic preciselymachined spacers. The two sapphires are then in contact with two Peltier elements and two thermistors that are connected to a controller able to maintain two distinct temperatures of the external sides of the sapphires with a rms stability of about $2 \mathrm{mk}$ over a day. More details about the experimental setup can be found in $[7,8]$.

Free-diffusion experiments have been performed by filling a quartz cylindrical cell first with the lighter component of the desired binary mixture and eventually letting the denser one slowly replace the lower half of the cell by means of a bended needle. No thermal control is utilized in the latter case other than letting the fluids stabilize at room temperature for one hour prior to injecting them in the cell. The vertical thickness of the cell is, in this case, $L=2 \mathrm{~cm}$. More experimental details are provided in previous publications $[13,14]$.

For all the experiments reported here, the optical technique of choice is the Shadowgraph except for one [14] in which the Schlieren setup was preferred. These two techniques share common physical principles and belong to the family of Near Field Scattering techniques [20-22]. The basic principle is that the light scattered by refractive index fluctuations is captured by a CCD camera close enough to the sample to interfere with the much more intense transmitted beam. In the special case of the Schlieren the beam is spatially filtered by an intensity mask (a blade) placed in the Fourier plane of a collecting lens prior to reaching the detector [23, 24]. Both techniques are similarly able to transform tiny fluctuations of the beam phase into fluctuations of the intensity that can be recorded by the CCD camera as sequences of images.

Intensity fluctuations are related to spatial and temporal variation of the refractive index of the sample that are originated by fluctuations of the temperature or the concentration depending on the optical contrast factors $[25,26]$. It is worth pointing out however that the performed dynamic analysis does not rely on these values for measuring the mass diffusion and Soret coefficients like most of the other existing optical techniques.
The acquired images are then analyzed by means of the Differential Dynamic Algorithm [7,8,13,14,27,28] allowing the extraction of the structure function of the fluctuations. This procedure has been described in detail in previous papers, we just mention here that due to the massive use of Fourier transforms in this analysis it is worth performing it by using parallel computing on a graphics processing unit (GPU) [28].

Due to the low frame rate of the utilized CCD cameras it is safe to consider that the contribution to the signal dynamics is due to concentration fluctuations only. Even for relatively rapid fluctuations in Soret-type experiments the thermal fluctuations decay much faster than the camera frame rate.

\section{Statics vs. dynamics}

Recently we proposed that the investigation of c-NEFs dynamics in a Soret-type experiment $[7,8]$ can be a powerful tool to obtain a measurement of the Soret and mass diffusion coefficients $\left(S_{T}\right.$ and $D$, respectively) of the analyzed binary mixture with an excellent agreement between our measurements and literature benchmark values [29]. To obtain the mass diffusion coefficient one has to investigate the dynamics of c-NEFs for wave vectors much larger than the $q_{s}^{*}$ while for the Soret coefficient the determination of the maximum time constant, i.e. the exact value of $q_{s}^{*}$ is needed. The extraction of these two values from data points is experimentally done by fitting them by means of eq. (5) with $D$ and $q_{s}^{*}$ as fitting parameters. One can equivalently try to fit the experimental static power spectrum by using eq. (6) with $I_{o}$ and $q_{s}^{*}$ as fitting parameters.

The aim of the present work is to compare the values of $q_{s}^{*}$ as experimentally determined by static $q_{s, \text { stat }}^{*}$ and dynamic $q_{s, \text { dyn }}^{*}$ analysis of c-NEFs. This comparison, shown in fig. 1, clearly indicates that the two values slightly, but systematically, differ by about ten per cent. This discrepancy is revealed in fig. 1 where the values of $q_{s, \text { stat }}^{*}$ obtained from static analysis are plotted against those of $q_{s, \text { dyn }}^{*}$ obtained from dynamic analysis. This plot includes all the measurements described in two of our recent papers $[7,8]$ of Soret-type experiments, as well as older data taken from refs. $[13,14]$ obtained by performing free-diffusion experiments. These data cover values of $q_{s}^{*}$ from about $75 / \mathrm{cm}$ up to $175 / \mathrm{cm}$ and are obtained by means of Schlieren (ref. [14]) or Shadowgraph (all the other reported experiments) setups. This is an important point as it allows us to exclude any impact of the Shadowgraph transfer function in the detection of the value of $q_{s, \text { stat }}^{*}$. The Schlieren technique, in fact, has a flat transfer function [24] thus making it much easier to evaluate the static scattering properties of the system, but at the expense of a much more demanding calibration of the experimental apparatus and a high sensitivity to tiny air convective motions. The latter is the main reason why Shadowgraph has been chosen for all the other experiments. 


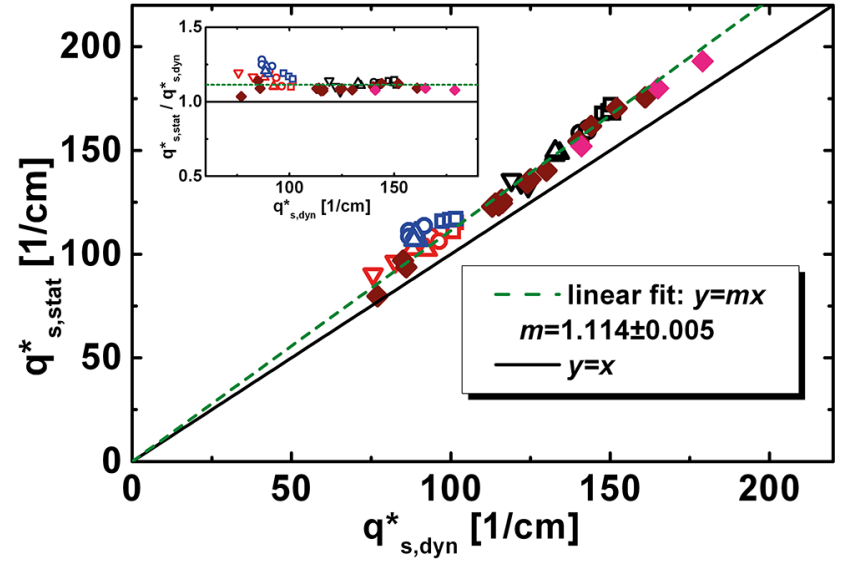

Fig. 1. (Color online) Solutal characteristic wave vector as obtained from static and dynamic analysis for different experiments. Open symbols denote the data from Soret experiments from refs. $[7,8]$, while filled symbols denote free-diffusion ones $[13,14]$. Color codes define the sample utilized: black is for THN-n-C12, red for THN-IBB, blue for IBB-n-C12, brown for glycerol/water against water from ref. [13] and pink for urea/water against water from ref. [14]. Symbols stand for the applied temperature differences: squares are for $\Delta T=20 \mathrm{~K}$, circles for $\Delta T=16 \mathrm{~K}$, up-triangles for $\Delta T=12 \mathrm{~K}$, downtriangles for $\Delta T=8 \mathrm{~K}$ and diamonds for the isothermal freediffusion experiments. Inset: ratio of the values of $q_{s}^{*}$ as obtained from the statics and dynamics vs. the one obtained from the dynamic analysis.

The graph reported in fig. 1 includes a linear regression of the experimental data points of the form $y=m x$, with $m$ as the only free parameter (dashed green line). For comparison, also $y \equiv x$ or $m=1$ is plotted (continuous black line). Within experimental error all data points are compatible with a linear fit with a coefficient $m=1.114 \pm 0.005$ thus indicating $11 \%$ systematic difference between the values for $q^{*}$ obtained from static and dynamic light scattering.

The inset of fig. 1 shows the same data by plotting the ratio of the values of $q_{s}^{*}$ as obtained from the statics and dynamics vs. the ones obtained from the dynamic analysis.

As shown in eq. (4) the product $q_{s}^{*} L$ to the fourth power is equivalent to the absolute value of the solutal Rayleigh number of the system. It is then worth investigating the impact of the static and dynamic light scattering measurements on its evaluation. In fig. 2 a comparison of the Rayleigh number as obtained from the static and dynamic evaluation of $q_{s}^{*}$ is reported. In this plot data points cover a wide range of solutal Rayleigh numbers from $10^{4}$ up to more than $10^{10}$. Also data deriving from Soret-type experiments are well separated from those relative to freediffusion experiments. This is due to the difference in the cell height which was $L=0.13 \mathrm{~cm}$ or $L=0.153 \mathrm{~cm}$ for Soret-driven experiments while it was $L=2 \mathrm{~cm}$ for the free-diffusion ones.

It is evident that, even if the experiments appear now very different from the point of view of the Rayleigh num-

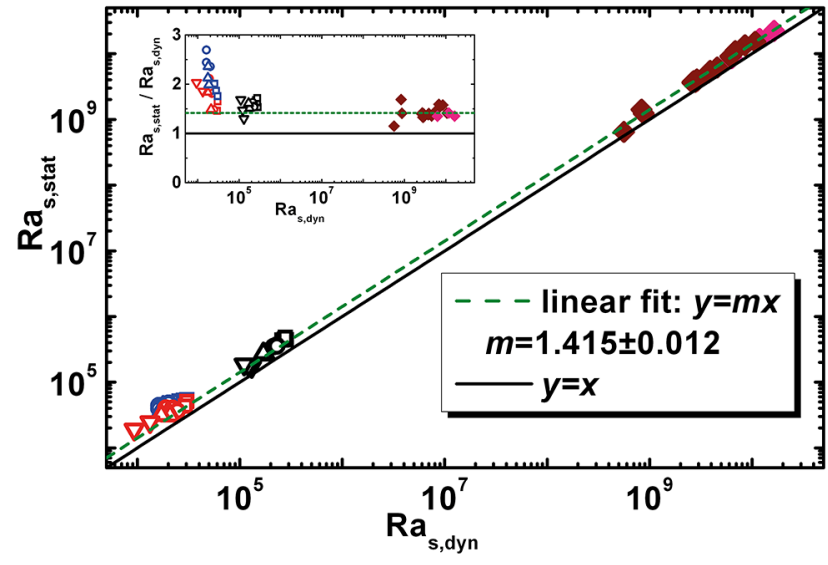

Fig. 2. (Color online) Log-log plot of the solutal Rayleigh number as obtained from static and dynamic analysis for different experiments. Symbols are the same as in fig. 1. Inset: ratio of the values of $R a_{s}$ as obtained from the statics and dynamics $v s$. the one obtained from the dynamic analysis.

bers, a discrepancy appears between the results from the dynamic and the static analysis. In fig. 2, too, a linear fit of the type $y=m x$ is shown (dashed green line) together with the case $m=1$ (continuous black line). In this case the two lines appear parallel because of the log-log representation. The fitting coefficient is then $m=1.415 \pm 0.012$ which is fully compatible with the previously obtained one if one takes into account the 4th power contained in eq. (4). The inset of fig. 2 shows the same data by plotting the ratio of the values of $R a_{s}$ as obtained from the statics and dynamics vs. the ones obtained from the dynamic analysis.

In our previous work we proposed the use of the dynamic analysis to evaluate the Soret coefficient of a binary mixture showing a very good agreement between the obtained values of the Soret coefficient and literature data. The Soret coefficient is actually proportional to the concentration gradient and thus to the Rayleigh number of the system. The result of this work is that if one attempts to do the same evaluation by means of static light scattering the concentration gradient is overestimated by roughly the $40 \%$, a quite remarkable difference!

We suggest that this discrepancy is relevant and points towards a fundamental problem in the theory of c-NEFs that has to be addressed in order to describe their statics with more accuracy. Actually in one recent paper [15] such an attempt was made by including the effect of confinement in the theory of c-NEFs. In the cited paper an expression for the statics of c-NEFs was not derived, but only computed. Moreover an expression was provided for its limit for vanishing wave vectors suggesting a reduction of the scattering intensity for very small wave vectors. However, this calculation predicts only a very small reduction in the "effective characteristic" wave vector $q_{s, \text { stat }}^{*}$. In fact in that paper a reduced value of the plateau for very small wave vectors was predicted which can explain 
a shift of the position of the "characteristic" wave vector $q_{s}^{*}$. We have estimated the impact of the more accurate theoretical calculation reported in [15] and the result is a reduction of the "effective" value of $q_{s, \text { stat }}^{*}$ of about one or two per cent, not sufficient to explain the effect reported in this work. However we point out that the calculations made in the mentioned paper took into account only very small (in modulus) values of the Rayleigh numbers $(-1500$, negative for Soret-type experiments) and we believe these calculations might not be accurate any more for the more realistic values used here ranging from $-10^{4}$ to $-10^{10}$. We also point out that even if the value obtained by the dynamic analysis appears to be sufficient for retrieving consistent values of the Soret coefficients for benchmark fluid mixtures, for very thin cells (unpublished data) the shape of the time constants for small wave vectors starts to deviate from the bell shape described by eq. (5) thus indicating that the confinement is also affecting the dynamics of c-NEFs. The latter contribution has never been discussed until now. For the cell thicknesses in the experiments reported here, however, we believe such an influence can be neglected and it does not affect the determination of $q_{s, \mathrm{dyn}}^{*}$.

\section{Conclusions}

In the present work we present evidence of a small, but systematic, difference between the values of the solutal "characteristic" wave vector as obtained from the dynamic $q_{s, \text { dyn }}^{*}$ or the static $q_{s, \text { stat }}^{*}$ light scattering analysis of cNEFs. As shown in previous papers, the value obtained from the dynamics allows us to obtain a correct determination of the Soret coefficient $S_{T}$. On the contrary, the value of $q_{s}^{*}$ obtained from the statics is systematically larger by more than ten per cent. In conclusion our results suggest that the available theory for static properties of c-NEFs is not able to provide a fully accurate description of concentration non-equilibrium fluctuations for large (negative) values of the Rayleigh numbers.

We thank José Maria Ortiz de Zárate, Alberto Vailati and Aleksandar Donev for fruitful discussions. We are also grateful to José Maria Ortiz de Zárate for critical reading of the paper. F.C. acknowledges financial support from the European Union under FP7 PEOPLE Marie Curie Intra European Fellowship, Contract No. IEF-251131, DyNeFI Project. This project has been financially supported by the Swiss National Science Foundation (Project Nr. 132736 and 149867).

\section{References}

1. J.M. Ortiz de Zárate, J.V. Sengers, Hydrodynamic Fluctuations in Fluids and Fluid Mixtures (Elsevier, New York, 2006).

2. J. Oh, J.M. Ortiz de Zárate, J.V. Sengers, G. Ahlers, Phys. Rev. E 69, 021106 (2004).

3. F. Balboa Usabiaga, J.B. Bell, R. Delgado-Buscalioni, A. Donev, T.G. Fai, B.E. Griffith, C.S. Pekin, Multiscale Model. Simul. 10, 1369 (2012).

4. A. Vailati, M. Giglio, Phys. Rev. E 58, 4361 (1998).

5. K. Balakrishnan, A.L. Garcia, A. Donev, J.B. Bell, Phys. Rev. E 89, 013017 (2014).

6. A. Donev, J.B. Bell, A. de la Fuente, A.L. Garcia, Phys. Rev. Lett. 106, 204501 (2011).

7. F. Croccolo, H. Bataller, F. Scheffold, J. Chem. Phys. 137, $234202(2012)$.

8. F. Croccolo, F. Scheffold, H. Bataller, C. R. Méc. 341, 378 (2013).

9. A. Vailati, R. Cerbino, S. Mazzoni, C.J. Takacs, D.S. Cannell, M. Giglio, Nat. Commun. 2, 290 (2011).

10. C.J. Takacs, A. Vailati, R. Cerbino, S. Mazzoni, M. Giglio, D.S. Cannell, Phys. Rev. Lett. 106, 244502 (2011).

11. A. Vailati, M. Giglio, Phys. Rev. Lett. 77, 1484 (1996).

12. A. Vailati, M. Giglio, Nature 390, 262 (1997).

13. F. Croccolo, D. Brogioli, A. Vailati, M. Giglio, D.S. Cannell, Phys. Rev. E 76, 41112 (2007).

14. F. Croccolo, D. Brogioli, A. Vailati, M. Giglio, D.S. Cannell, Appl. Opt. 45, 2166 (2006).

15. J.M. Ortiz de Zárate, J.A. Fornés, J.V. Sengers, Phys. Rev. E 74, 046305 (2006).

16. R.D. Mountain, J.M. Deutch, J. Chem. Phys. 50, 1103 (1969).

17. C. Giraudet, H. Bataller, Y. Sun, A. Donev, J.M. Ortiz de Zárate, F. Croccolo, submitted, http://arxiv.org/abs/1410.6524.

18. P.N. Segrè, J.V. Sengers, Physica A 198, 46 (1993).

19. P.N. Segrè, R.W. Gammon, J.V. Sengers, Phys. Rev. E 47, 1026 (1993).

20. S. Trainoff, D.S. Cannell, Phys. Fluids 14, 1340 (2002).

21. R. Cerbino, A. Vailati, Curr. Opin. Colloid Interface Sci. 14, 416 (2009) http://dx.doi.org/10.1016/j.cocis. 2009.07.003.

22. F. Giavazzi, R. Cerbino, J. Opt. 16, 083001 (2014) doi: 10.1088/2040-8978/16/8/083001.

23. G.S. Settles, Schlieren and Shadowgraph Techniques (Springer, Berlin, 2001).

24. F. Croccolo, D. Brogioli, Appl. Opt. 50, 3419 (2011).

25. F. Croccolo, M.A. Arnaud, D. Bégué, H. Bataller, J. Chem. Phys. 135, 034901 (2011).

26. F. Croccolo, F. Plantier, G. Galliero, G. Pijaudier-Cabot, M.Z. Saghir, F. Dubois, S. Van Vaerenbergh, F. Montel, H. Bataller, Rev. Sci. Instrum. 82, 126105 (2011).

27. R. Cerbino, V. Trappe, Phys. Rev. Lett. 100, 188102 (2008).

28. G. Cerchiari, F. Croccolo, F. Cardinaux, F. Scheffold, Rev. Sci. Instrum. 83, 106101 (2012).

29. J.K. Platten, M.M. Bou-Ali, P. Costesèque, J.F. Dutrieux, W. Köhler, C. Leppla, S. Wiegand, G. Wittko, Philos. Mag. 83, 1965 (2003). 